\title{
Breakfast characteristics, perception, and reasons of skipping among 8th and 9th- grade students at governmental schools, Jenin governance, West Bank
}

\author{
Manal Badrasawi* (D), Ola Anabtawi and Yaqout Al-Zain
}

\begin{abstract}
Background: There is growing recognition of the important role of breakfast in children's nutrition, and the potential harms related to skipping breakfast, including its contribution to obesity and non-communicable diseases. The patterns associated with skipping breakfast may be related to the nutrition transition. This study aimed at exploring the composition of breakfast consumed by Palestinian school children and their perceptions toward this meal. It also aimed at exploring skipping breakfast prevalence, reasons, and its association with selected schoolchildren's sociodemographic variables and behavioral patterns.

Methods: A cross-sectional online survey was conducted among 12- to 14-year-old schoolchildren from 4 governmental schools in urban and rural areas of the Jenin district in Palestine. The questionnaire included information about children and parents sociodemographic and behaviours, breakfast patterns and composition, reasons for skipping breakfast, and items on schoolchildren's perception toward breakfast. Statistical analysis of the relevant factors was undertaken using SPSS software.

Results: In a sample of 193 schoolchildren, only 32\% reported consuming breakfast all year round. The main reasons reported for skipping breakfast were not feeling hungry, not having the time, and lack of appetite. The vast majority (79\%) believed breakfast was beneficial for general health. Sleeping before $10 \mathrm{pm}$, regular exercise ( $p$ value $<0.05$ ). and shorter screen time were all significantly associated with a higher level of breakfast consumption ( $p$ value $<0.01$.

Conclusion: Understanding the reasons for missing or skipping breakfast factors which make skipping it more likely, should inform public health strategies to promote breakfast consumption. For example, our findings suggest that awareness of the importance of breakfast was not a significant contributor to skipping breakfast, compared to other structural and cultural factors.
\end{abstract}

Keywords: Breakfast, Breakfast skipping, Perception, Palestine

\footnotetext{
* Correspondence: m.badrasawi@najah.edu

Nutrition and Food Technology Department, Faculty of Agriculture and Veterinary Medicine, An-Najah National University, Tulkarm, West Bank PO. Box 7, Nablus, Palestine
}

(c) The Author(s). 2021 Open Access This article is licensed under a Creative Commons Attribution 4.0 International License, which permits use, sharing, adaptation, distribution and reproduction in any medium or format, as long as you give appropriate credit to the original author(s) and the source, provide a link to the Creative Commons licence, and indicate if changes were made. The images or other third party material in this article are included in the article's Creative Commons licence, unless indicated otherwise in a credit line to the material. If material is not included in the article's Creative Commons licence and your intended use is not permitted by statutory regulation or exceeds the permitted use, you will need to obtain permission directly from the copyright holder. To view a copy of this licence, visit http://creativecommons.org/licenses/by/4.0/ The Creative Commons Public Domain Dedication waiver (http://creativecommons.org/publicdomain/zero/1.0/) applies to the data made available in this article, unless otherwise stated in a credit line to the data. 


\section{Introduction}

A lot has been researched, written and published about the nutritional transition in low and middle income settings [1]. This transition includes physical activity shifting towards more sedentary lifestyles, and changing dietary habits. In Palestine, such new dietary habits include an increased consumption of saturated fats, reduced fruit and vegetable intake, and reduced fibre intake. They also include an increasing prevalence of skipping breakfast [2]. This transition has been shown to be a significant risk factor for increased noncommunicable diseases. Studies show that individuals who skip breakfast are at increased risk of high BMI and type - 2 diabetes $[3,4]$, which are already at high and increasing rates in Palestine $[5,6]$. Globally, skipping breakfast is highest among adolescents, who are consequently at risk of poorer academic performance and physical growth [7]. This makes this issue vitally important in the Palestinian context where one-quarter of the population is made up of adolescents [8].

The most recent comprehensive study studying health behaviours among adolescents was conducted as part of WHO's Health Behaviour in School-age Children (HBSC) study in 2004 [9]. The study used a crosssectional survey to investigate health behaviours among adolescents in 35 countries, including the West Bank and Gaza Strip in Palestine. The survey identified several health concerns, including that $45 \%$ of adolescents did not consume breakfast, as well as its link to other health behaviours. Several studies since then have considered breakfast consumption among adolescents with similar findings to the HBSC study, in 2009 and 2010 in Palestine, and many other studies in other Arab countries [10-12]. None of these studies, including the HBSC study, considered the underlying reasons for skipping breakfast or people's perceptions toward it in Palestine.

There are significant and justified concerns about the rising rates of NCDs and obesity in Palestine. Addressing their risk factors necessitates understanding the causes and people's perceptions of health behaviours which interventions seek to promote [13]. In the case of breakfast skipping, for example, there is a good understanding of the problem, but not of its causes or people's perceptions toward breakfast meal. Therefore, a deep and compressive understanding of skipping breakfast helps come out with appropriate interventions seeking to promote breakfast as a positive health behaviour. The aim of this study is, to explore breakfast consumption in a representative sample of children aged 12-14 years. The objectives are first to understand the associations of skipping breakfast with age, gender, parental education level, and sleeping time, as potential determinants of skipping breakfast. Second, to explore the associations between the reasons for skipping breakfast and perceptions towards breakfast consumption. Third, to investigate breakfast characteristics in association with the previous objectives.

\section{The methodology and study instruments}

This study used a cross-sectional design to answer the research objectives. The participants were grades eight and nine schoolchildren selected from four governmental schools in Jenin District in the West Bank, Palestine. The four schools included two girls' and two boys' schools, randomly selected from urban and rural areas. A formal letter was sent to the Ministry of Education to get permission for the data collection from the selected schools.

\section{Data collection}

Data collection started in October 2020 and ended in November 2020. The final version of the questionnaire described above was formulated into an online survey. The first step of the data collection was schools selection; 4 schools from urban and rural areas of Jenin governance were invited by the Ministry of Education. The distribution of the link for the online data sheet started after the verbal consent of participation was taken from the school principals and the parents of schoolchildren. The students were invited to fill in the online forms through their teachers by sending the link through the e-classes and through the schools' webpages. The students were briefed on how to fill out the online questionnaire by their teachers and they were asked to sign the consent form of participation online. The students were asked to report the correct data regarding their personal information and they were told to answer the questions individually. They were also informed that there were no wrong or correct answers and the data would be used only for research purposes.

\section{Ethical approval}

The Palestinian Ministry of Education approved the study protocol once it had been reviewed by the Research and Quality Control committee. The study protocol was also approved by the Institution Review Board (IRB) ethical committee at An-Najah National University. Having got the approval, an invitation letter was sent to the selected schools through the Directorate of Education- Jenin office. As the participant's age was less than 18 years old, the consent of participation was taken first from the school principals, then from the parents through the school teachers. Informed consent was obtained from all the parents before the data collection. The online questionnaire was sent to the students through their teachers in the selected schools after the teachers got the parents' agreement. All students were informed that their participation was not compulsory 
and the data would be used only for research purposes. They were asked to answer the questions individually.

\section{Sample size and sampling procedures}

Using $\mathrm{G}$ power software and an alpha of 0.05 (two-sided) and $80 \%$ power, sample size calculations revealed that a minimum of 175 participants were required to determine the prevalence of breakfast skipping. Then the sample size was calculated again to determine the association between breakfast skipping and other variables. The mean difference test was selected, $5 \%$ level of significance, $(80 \%)$ power. The required sample size was 208 participants. The inclusion criteria were: all students who are in grades 8th and 9th from Jenin governmental schools in Jenin are willing to participate and have completed the online questionnaire. The students, who did not answer the primary questions i.e. questions related to breakfast, reasons for skipping and perceptions toward breakfast, were excluded from the final data analysis. A total of 1250 students from grades 8th and 9th from 4 different schools were invited to fill in the online questionnaire. Only 203 students responded to the invitation and gave the required data, with a $16 \%$ responding rate. Ten (10) students were excluded due to missing data; and only 193 students were included in the final analysis.

\section{Research instrument}

The questionnaire was developed based on a thorough literature review pertaining to breakfast consumption among school students from different age groups. Two specialists in nutrition prepared the initial questionnaire items using the participants' native language (i.e. Arabic). Content validity was examined by five (5) experts in nutrition and three (3) in assessment and research methods. A few items were amended based on the experts' comments and suggestions. Other items were deleted and replaced with new ones (i.e. items related to reasons for breakfast skipping, mainly the repeated or unclear ones) to ensure that all items measure the investigated construct accurately. The revised questionnaire was sent for Arabic language editing before it was distributed. The final version of the questionnaire consisted of three sections: section one was for socio demographic characteristics of the students, including gender, area of living, family type, number of family members, parents' education, parents' work status and pocket money. The second section included the medical history, lifestyle and presence of chronic diseases. The lifestyle variables included sleeping hours, sleeping time, wake up time, methods of going to school (walking or using transport), going for exercise and screen time. The third section had breakfast related data: 3 items about breakfast consumption during school and weekend days (answers: always if students eat breakfast 6-7 days/week, sometimes if students eat breakfast less than 5 times or less per week, never if students do not eat breakfast at all) in addition to consuming meals at schools, two openended questions about the types of food consumed in breakfast and school meals, eight dichotomous questions with three point Likert scale: agree, no opinion and disagree. The reliability test was done using Chronbach alpha test for each section separately. The reliability for the reasons for skipping breakfast was 0.81 , and the reliability for perceptions was 0.59 for the seven items; however, the reliability for the six items was improved by deleting item no. 7 .

\section{Statistical analysis}

The Statistical package for the social Sciences SPSS, version 21, was used to analyze the collected data. Descriptive analysis including means and standard deviations were used to analyze data pertaining to continuous dependent and independent variables. The categorical data was described by percentages. Independent $t$-test and ANOVA tests were conducted to examine the differences between selected independent variables, while the Chi Square test was employed to examine the association between the categorical independent variables and the nominal levels, with a significance level of 0.05 .

\section{Results}

\section{Characteristics of the participants}

A total of 193 students were included in the final analysis. The majority of the sample are girls (70.5\%), eighth grade $(57 \%)$, and live in a nuclear family $(88.1 \%)$. The number of family members ranged from 3 to 15 members; $18.2 \%$ of the families have $\leq 5$ members, $70 . \%$ have (6-8 members) and $11.5 \%$ of the family members are 9 and above. The means of pocket money as reported by the participants is $5.4 \pm 3.2$, NIS-New Israeli Shekel -/day. In regard to lifestyle, (83.7\%) of the students walk to school every day and only (19.3\%) go for exercise regularly. The mean of screen time is $4.4 \pm 2.8 \mathrm{~h} /$ day. The mean of sleeping hours is $8.1 \pm 1.4$, hour/day.

\section{Prevalence of breakfast consumption and meal characteristics}

Figure 1 shows the prevalence of breakfast consumption on school days, weekends and all days. The majority of participants $(62.2 \%)$ reported eating breakfast on weekends, followed by school days (37.3\%) and all days (32.1\%). While the prevalence of breakfast skipping was higher in school days as compared to weekends. In regard to taking meals to school, $80.3 \%$ of the students said they take meals to school every day, while the rest said they do not take meals to school. 


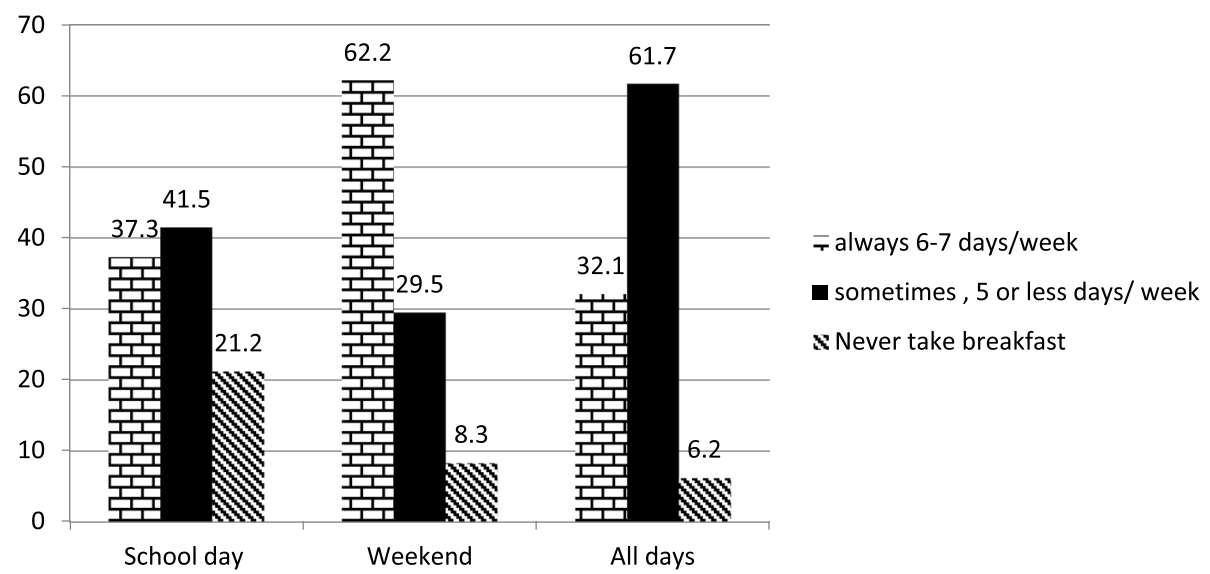

Fig. 1 Breakfast and school meal consumption during school and weekend days

Breakfast consumption and associated factors (sociodemographic and lifestyle)

Table 1 shows the association between breakfast consumption with sociodemographic variables and lifestyle. Using the chi square test, the results show that there is no relationship between breakfast consumption in general, schooldays, or on weekends, gender, grade, family type, and parents 'education, or parents' work status. However, the every day breakfast consumption is associated with living in cities; and never consuming breakfast is associated with living in villages, $p<0.05$ using the Chi Square test. Moreover, the relationships between breakfast consumption with the number of family members and the amount of pocket money are not significant using one way ANOVA test. In regard to lifestyle factors, early sleeping i.e., before 10:00 pm is significantly associated with a higher prevalence of breakfast consumption, $p<0.05$, using the Chi Square test. Similarly, a significant association is found between going for exercise (regular, irregular) with a higher prevalence of every day breakfast consumption, $p<0.05$, using the Chi Square test, whereas the other lifestyle variables (walking to school, waking up early) show a non-significant association with breakfast consumption. For continuous variables in the lifestyle (i.e. hours of night sleeping and hours of screen time), the results show a significant relationship between breakfast consumption and screen time; students who eat breakfast every day reported shorter screen time $(3.3 \pm 2.3)$ hours/ day, students who sometimes eat breakfast $(4.66 \pm 3.1)$ hours compared to who always skip breakfast $(5.4 \pm 2.7)$ hours/ day, $p<0.01$ using one way ANOVA test. Similar trend is shown for hours of night sleep; students who skip their breakfast reported shorter night sleep $(7.2 \pm 2)$ compared to students who always or sometimes eat breakfast with $(8.1 \pm$ 1.1 and $8.1 \pm 1.3$ ) respectively, with no significant differences using one way ANOVA test, $p>0.05$.

\section{Breakfast meal characteristics}

Table 2 shows the breakfast and school meal components. Sandwiches are the most consumed type of meal in both breakfast and school meals. Breakfast is mostly prepared by the parents; and students eat their breakfast with their siblings $(41.1 \%)$, and with their whole family (34.4\%).

\section{Breakfast skipping reasons and associated factors}

Table 3 shows the reasons for breakfast skipping as reported by 129 students who always or sometimes skip breakfast. The most common reason for skipping breakfast is that "they don't feel hungry in the morning," followed by "they don't have time to eat" and "they don't like to eat early," while "they don't like the food" and "they want to lose weight" are the least common. The results reveal that there is an association between female gender and the eight reasons for breakfast skipping, $p>$ 0.05 using the Chi Square test. The same finding is found with school location, grade, students living area and family type. For parents' education, there is a significant association between item 5 (I don't find ready food to eat) with lower parents' education level, $p<0.05$ using the chi square test. The same significant association was found with parents' working status (both parents are not working), $p<0$ 0.05.Likewise, item.7 (My family skips breakfast and so $I d o$ ), is associated with both parents are not working, $p<0.01$ using the Chi square test.

\section{Perceptions toward breakfast and associated factors}

As shown in Table 4, students reported different perceptions toward breakfast. Breakfast consumption is very important for general health $(79.3 \%)$ and for good cognitive performance $(81.3 \%)$, while $64 \%$ disagree that it increases weight, (69.9\%) disagree that it causes gastrointestinal disturbances, and $81.3 \%$ disagree that it makes them feel lazy and less energetic. No significant 
Table 1 Participants' breakfast consumption according to sociodemographics, medical history and lifestyle characteristics presented in $n(\%) /$ mean $\pm \mathrm{sd}$

\begin{tabular}{|c|c|c|c|c|c|}
\hline & \multirow[t]{2}{*}{ Total N (\%) } & \multicolumn{3}{|c|}{ Breakfast consumption } & \multirow[t]{2}{*}{$P$ value } \\
\hline & & All days & Sometimes & Never & \\
\hline \multicolumn{6}{|l|}{ Socio demographic } \\
\hline \multicolumn{6}{|l|}{ Gender } \\
\hline Boys & $57(29.5)$ & $18(31.6)$ & $32(56.1)$ & $7(12.3)$ & \multirow[t]{2}{*}{0.527} \\
\hline Girl & $136(70.5)$ & $44(32.4)$ & $67(49.3)$ & $25(18.4$ & \\
\hline \multicolumn{6}{|l|}{ School location } \\
\hline City & $103(53.4)$ & $40(38.8)$ & $51(49.5)$ & $12(11.7)$ & \multirow[t]{2}{*}{$0.039 * 1$} \\
\hline Village & $90(46.6)$ & $22(24.4)$ & $48(53.3)$ & $20(22.2)$ & \\
\hline \multicolumn{6}{|l|}{ Grade } \\
\hline 8th & $110(57)$ & $40(36.4)$ & $55(59)$ & $15(13.6)$ & \multirow[t]{2}{*}{0.24} \\
\hline 9th & $83(43)$ & $22(26.5)$ & $44(53)$ & $17(20.5)$ & \\
\hline \multicolumn{6}{|l|}{ Family type } \\
\hline Nuclear family & $170(88.1)$ & $52(30.6)$ & $89(52.4)$ & $29(17.1)$ & \multirow[t]{2}{*}{0.459} \\
\hline Extended family & $23(11.9)$ & $10(43.5)$ & $10(43.5)$ & $3(13)$ & \\
\hline \multicolumn{6}{|l|}{ Parents' education } \\
\hline Both parents have primary education & $20(10.4)$ & $4(20)$ & $14(70)$ & $2(10)$ & \multirow[t]{6}{*}{0.064} \\
\hline Both parents have secondary education & $31(16.1)$ & $15(48.4)$ & $(11(35.5)$ & $5(16.1)$ & \\
\hline Both parents have university degree & $40(20.7)$ & $12(30)$ & 23 57.5) & $5(12.5)$ & \\
\hline Mixed level; primary /secondary & $34(17.6)$ & $16(47.1)$ & $14(41.2)$ & $4(11.8)$ & \\
\hline Mixed level; primary /university degree & $43(22.3)$ & $10(23.3)$ & $25(58.1)$ & $5(18.6)$ & \\
\hline Mixed level; secondary /university degree & $25(13)$ & $5(20)$ & $12(48)$ & $8(32)$ & \\
\hline \multicolumn{6}{|l|}{ Parents working status } \\
\hline Both parents are not working & $5(2.6)$ & $2(40)$ & $3(60)$ & $0(0)$ & \multirow[t]{3}{*}{0.579} \\
\hline Both parents are working & $47(24.4)$ & $12(25.5)$ & $28(59.6)$ & $7(14.9)$ & \\
\hline One of parents' work & $140(72.5)$ & $48(34)$ & $68(48.2)$ & $25(17.7)$ & \\
\hline \multicolumn{6}{|l|}{ Medical history } \\
\hline \multicolumn{6}{|l|}{ Presence of Chronic diseases } \\
\hline Yes & $19(9.8)$ & $8(42.1)$ & $8(42.1)$ & $3(15.8)$ & \multirow[t]{2}{*}{0.604} \\
\hline No & $174(90.2)$ & $54(31)$ & $91(52.3)$ & $29(16.7)$ & \\
\hline \multicolumn{6}{|l|}{ History of surgical operation } \\
\hline Yes & $8(4.1)$ & $4(50)$ & $3(37.5)$ & $1(12.5)$ & \multirow[t]{2}{*}{0.541} \\
\hline No & $185(95.9)$ & $58(31.4)$ & $96(51.9)$ & $31(16.8)$ & \\
\hline \multicolumn{6}{|l|}{ Lifestyle } \\
\hline \multicolumn{6}{|l|}{ Go to school } \\
\hline Walking & $159(83.7)$ & $48(30.2)$ & $83(52.2)$ & $28(17.6)$ & \multirow[t]{2}{*}{0.335} \\
\hline Using transports (public or private) & $31(16.3)$ & $13(41.9)$ & $15(48.4)$ & $3(9.7)$ & \\
\hline Go for exercise (home, outside) & & & & & \\
\hline Yes regularly & $37(19.3)$ & $17(45.9)$ & $17(45.9)$ & $3(8.1)$ & 0.015 \\
\hline Yes, irregular & $109(56.8)$ & $35(32.1)$ & $59(54.1)$ & $15(13.8)$ & \\
\hline No & $46(24)$ & 9 (19.6) & $23(50)$ & $14(30.4)$ & \\
\hline Wake up time & & & & & \\
\hline Before 6:00 am & $24(12.9)$ & $8(33.3)$ & $9(37.5)$ & $7(29.2)$ & 0.09 \\
\hline 6:00-7:00 am & $98(52.7)$ & $37(37.8)$ & $49(50)$ & $12(12.2)$ & \\
\hline
\end{tabular}


Table 1 Participants' breakfast consumption according to sociodemographics, medical history and lifestyle characteristics presented in $\mathrm{n}(\%) /$ mean $\pm \mathrm{sd}$ (Continued)

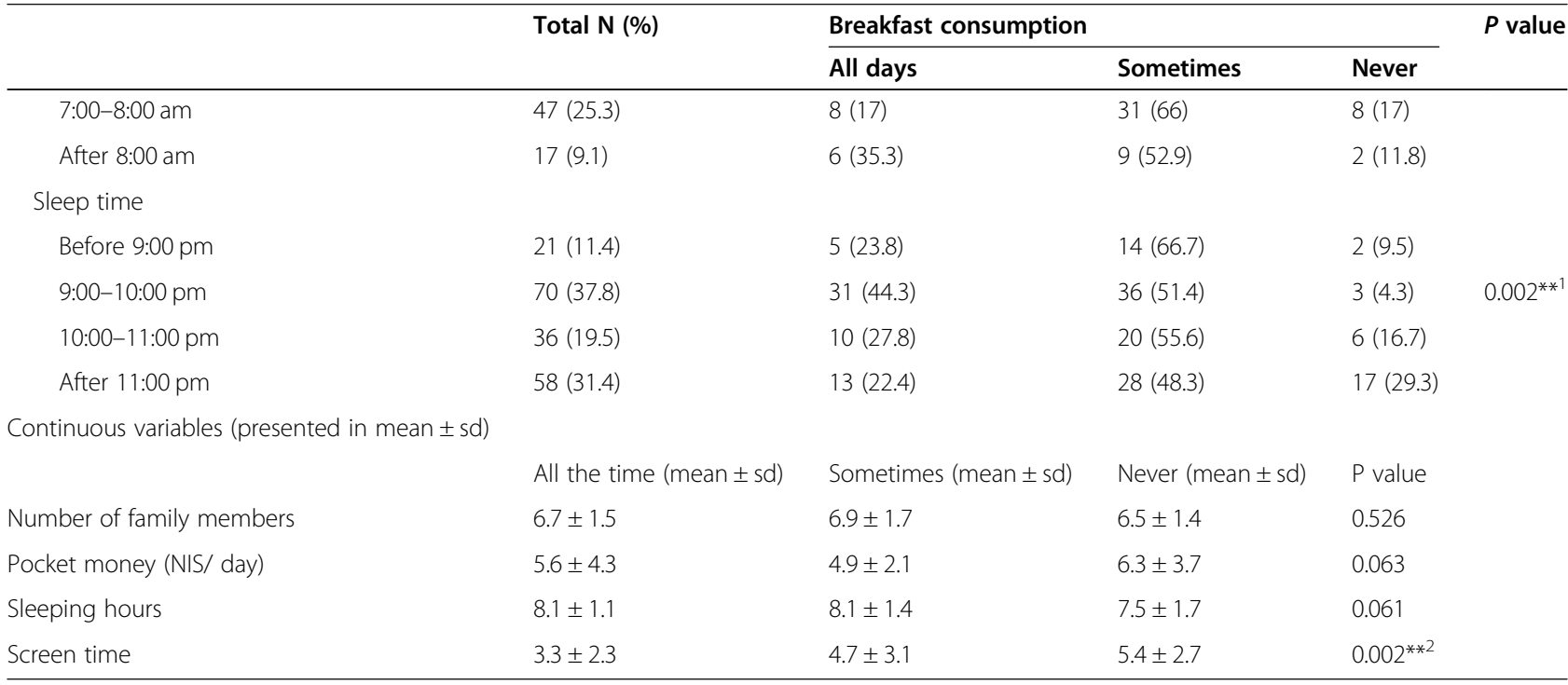

*Significant at $p<0.05, * *$ significant $p<0.01$

${ }^{1}$ using chi square test, ${ }^{2}$ using one way ANOVA

Table 2 Breakfast and school meal characteristics and consumption pattern presented in $\mathrm{n}(\%)$

n $\%$

Breakfast characteristics (school days)
Type of food
Sandwich (cheese, labaneh, Zaater, sausage)
Milk+ cookeies (cake +biscuits)
Tea + cookies (cake +biscuits)
Milk+ cereals
Traditional breakfast
Consumption company
Alone
With siblings
With family
Breakfast preparation
Parents / mothers
Ready food from outside the house
I prepare it myself
Brothers or sisters

\section{School meal characteristics}

Type of food

Sandwich (Cheese, labaneh, chocolate)

Sandwich + fruits

Sandwich and juices

Cake or cookies, chips

Fruits only

Sandwich+ cookies or chocolate or cake relationship was found between students' perceptions and any of the socio demographic variables: gender, school locations, parents' education or parents' working status, $p>0.05$.

\section{Discussion}

Findings of this study indicate that only $32 \%$ of children consume breakfast daily all-year round, and $62 \%$ consume breakfast daily during the weekend. Previous studies with similar samples conducted in Palestine reported $50 \%$ breakfast consumption twice or fewer per week [10], 45\% breakfast consumption 4 days-per-week during school term [14], and $62 \%$ breakfast consumption [11]. The latest study with a similar sample from a neighbouring country, Jordan reported $80 \%$ of children consuming breakfast [15]. In Saudi Arabia, a survey recently reported that $79 \%$ of children skipped daily breakfast but

Table 3 Students (Yes) answer for reasons for breakfast skipping

\begin{tabular}{lll}
\hline Reasons & $\mathbf{n}$ & $\%$ \\
\hline I don't feel hungry & 77 & 59.7 \\
No time to have breakfast & 65 & 50.4 \\
I don't like to eat early & 63 & 48.8 \\
I feel uncomfortable when I eat & 42 & 32.6 \\
I don't find ready food to eat & 25 & 19.4 \\
I need to lose weight & 20 & 15.5 \\
My family skip the breakfast and so I do & 30 & 23.3 \\
I don't like the food choices & 19 & 14.7 \\
\hline
\end{tabular}


Table 4 Students' positive and negative perceptions towards breakfast consumption

\begin{tabular}{|c|c|c|}
\hline Perceptions & $\mathrm{n}$ & $\%$ \\
\hline \multicolumn{3}{|c|}{ I believe breakfast consumption is very important to general health } \\
\hline Agree & 153 & 79.3 \\
\hline No opinion & 20 & 10.4 \\
\hline Disagree & 20 & 10.4 \\
\hline \multicolumn{3}{|c|}{$\begin{array}{l}\text { I believe breakfast consumption increase the concentration and } \\
\text { memorization during the classes }\end{array}$} \\
\hline Agree & 157 & 81.3 \\
\hline No opinion & 19 & 9.8 \\
\hline Disagree & 17 & 8.8 \\
\hline \multicolumn{3}{|c|}{ I believe breakfast consumption increase the weight } \\
\hline Agree & 25 & 13.0 \\
\hline No opinion & 44 & 22.8 \\
\hline Disagree & 124 & 64.2 \\
\hline \multicolumn{3}{|c|}{$\begin{array}{l}\text { I believe breakfast consumption may leads to gastrointestinal } \\
\text { disturbances }\end{array}$} \\
\hline Agree & 13 & 6.7 \\
\hline No opinion & 45 & 23.3 \\
\hline Disagree & 135 & 69.9 \\
\hline \multicolumn{3}{|c|}{ I believe breakfast consumption make me feel lazy and less energeti } \\
\hline Agree & 19 & 9.8 \\
\hline No opinion & 17 & 8.8 \\
\hline Disagree & 157 & 81.3 \\
\hline \multicolumn{3}{|c|}{$\begin{array}{l}\text { I believe I can make up the breakfast with other meals during the da } \\
\text { without any difference on health }\end{array}$} \\
\hline Agree & 57 & 29.5 \\
\hline No opinion & 33 & 17.1 \\
\hline Disagree & 103 & 53.4 \\
\hline
\end{tabular}

weekends compared to weekdays [12]. Our findings indicate a lower proportion of children consuming breakfast in Palestine than in neighbouring countries.

Breakfast consumption was not associated with gender, age, parents' education, parents' work status, or pocket money, but was positively associated with living in a village. Breakfast consumption was significantly associated with regular exercise, sleeping before $10 \mathrm{pm}$, and shorter screen time. Previous studies in the same region found different associations. For example, an association between female gender and breakfast consumption has been observed [10, 11, 16]. However, this association is not seen in Saudi Arabia or Jordan $[12,15]$. A relationship between education and breakfast consumption has also been seen, with a higher parental education level predicting lower breakfast skipping [10, $12]$, and a higher paternal level of education predicting lower breakfast skipping [14]. In 2009, no relationship was seen between rural or urban living and breakfast consumption [11]. The same study also found an association between breakfast consumption and socioeconomic status.

Almost $70 \%$ of children in the current study had more than $2 \mathrm{~h}$ of screen time per day, with a higher proportion among children, and 66\% did not sleep the recommended amount, both of which were associated with reduced breakfast consumption [17]. Other studies have found similar associations, with waking up late associated with skipping breakfast [18], and longer sleep associated with decreased breakfast skipping [19].

Previous surveys on a regional level have not studied the relationship between breakfast consumption and exercise. Regular breakfast consumption was associated with health-promoting behaviours including exercise [20], and adolescents who skip breakfast are less likely to exercise regularly [21].

Of those consuming breakfast at home, 35\% ate sandwiches (white cheese, labaneh, zaatar), $8 \%$ ate cereal and milk, and $18 \%$ cookies with milk or tea. $40 \%$ had breakfast prepared by their parents. A study conducted in Qatar, surveying a younger age group, found that $90 \%$ ate eggs or cheese for breakfast, and $42 \%$ sweets and chocolate [22]. In a study in Saudi Arabia, $48 \%$ had fried eggs sandwiches, $46 \%$ had breakfast cereal, and $41 \%$ had spread cheese sandwiches. In the same sample, $80 \%$ had breakfast prepared by parents, and only $4-7 \%$ consumed more healthy options (such as labaneh or zaatar sandwiches) [17].

\section{Reasons for skipping breakfast}

In our sample, the most common reasons given for skipping breakfast were not feeling hungry $(60 \%)$, not having time (50\%), and not enjoying eating early (49\%). Other reasons included a desire to lose weight $(15 \%)$ and the rest of the family skips (23\%).There was a statistically significant association between being female and all the aforementioned factors. Both parents being out of work and lower education level were significantly associated with children reporting not having anything to eat (20\%). These findings are similar to reasons identified in other studies in Arab countries and globally. In a study in Jordan, the top reasons were poor appetite (65\%), not having time $(60 \%)$, having nothing to eat $(60 \%)$, and having no one to prepare breakfast (58\%) [15]. In Saudi Arabia, $48 \%$ reported not feeling hungry and $36 \%$ reported not having time [17]. In an Australian study, the two most common reasons were a lack of hunger and a lack of time [23].

\section{Perceptions for breakfast}

There were generally positive perceptions of breakfast among our sample, with $79 \%$ believing it is beneficial for general health, $81 \%$ agreeing that it increases 
concentration and memorisation at school, only $13 \%$ believing that it contributes to weight gain, and $81 \%$ disagreeing that breakfast consumption made them lazy and less energetic. Such perceptions have not been found in previous research in the Arab world. There have been studies investigating perceptions of breakfast elsewhere in the world, but with very different samples and methodologies [24-26].

\section{Implications}

Many studies have investigated patterns of breakfast consumption and its associations with various individual and societal factors. This study has delved deeper into the reasons behind skipping breakfast, and children's perceptions toward breakfast. This study examined factors which have not previously been investigated in Palestine, such as the relationship of children's breakfast consumption with exercise, sleep, and screen time. These associations are particularly important because they form part of the nutritional transition.

The current research findings have led to important public health implications. For example, perception of breakfast is generally positive, with a high level of awareness of its importance and role in improving performance at school and health in general. Public health campaigns educating children on the importance of breakfast are therefore unlikely to be valuable. For a small subset of children, particularly girls, perception of weight may, on the other hand, be an important factor to address. This is particularly important in light of the increasing rates of eating disorders in Palestine.

It is essential to note that, currently in Palestine, there are no policies in place to mandate providing breakfast at schools for any age group in any class. There are only strategies and policies to control the type of food supplied into school canteens. This includes banning specific high-fat processed foods (i.e. chips and crisps). On a local level, there are some private initiatives to provide home-made food to schools.

The lack of association between breakfast consumption and parents' work status was a surprising finding, as higher education and work-status are normally associated with increased healthy behaviour and have been found to be positively associated with breakfast consumption in previous studies in Palestine and neighbouring countries. Yet, important reasons for skipping breakfast included having no one to prepare breakfast and skipping breakfast because the rest of the family does. It is vital to understand the context defining public health issues to facilitate the design and implementation of effective public health interventions. In relation to breakfast consumption, for example, it is important to appreciate the nutrition transition, and it's various components, such as sedentary lifestyles, increased focus on weight, higher exposure to technology, and higher female employment rates. One potential intervention to improve breakfast consumption in Palestine may be to promote breakfast provision in schools. However, this would need a more comprehensive exploration of the characteristics of breakfast for different age groups and backgrounds. This was touched upon in our survey but would need more detailed investigation. In addition, the focus should be on further examining the reasons for skipping breakfast, nutritional education to eliminate misperceptions about breakfast, and correction of unsuitable nutritional components of breakfast.

\section{Strengths and limitations \\ Strengths}

This study sheds light on factors related to breakfast consumption which are vital to designing public health interventions. These include a detailed investigation of the different associations between breakfast consumption and different characteristics of children and their parents, as well as factors which have not been looked at in the Palestinian context, such as exercise, sleep, and screen time. The survey also offered valuable insight into perceptions of breakfast and reasons for skipping it. In addition, the sample we surveyed is representative of the selected studied age group population.

\section{Limitations}

There was a relatively low response rate to the survey, which may challenge the generalisability of the findings, despite the representative sample. Although it is uncommon for studies with the same population in the neighboring countries to have this low response rate, $[15,27]$ it is important to consider that the main reason for this low rate might be using online survey due to the covid19 pandemic, which has made it difficult meet school children face to face. Within the survey, there was possible overlap in the definition of breakfast between the meal consumed at home before school, and the one consumed at school in the morning. This may have had an impact both on the responses and their analysis. Finally, although we collected basic information on the characteristics of breakfast which children consumed, this was an open-ended qualitative question. This means that there was insufficient detail to quantify what children consumed.

\section{Conclusion}

Breakfast is well-recognised as an important meal in our sample of Palestinian 8th and 9th school children. However, these schoolchildren are not consuming breakfast consistently due to several reasons or obstacles. On an individual level, these include long screen time and short sleep. On a family level, household factors, such as not 
having anyone to prepare food, and perceptions or fears around weight are important determinants. Public health interventions would therefore have higher chances of success if targeted at addressing such factors, rather than simply promoting breakfast. Especially that students of this sample have shown good or positive perception towards breakfast consumption.

Future research should aim to better understand the culture around breakfast, such as what children eat, and where they would be prepared to eat it, to support the design of effective public health interventions around it.

\section{Acknowledgments}

We would like to acknowledge the lecturers who helped the researchers with the data collection. We would like to express our gratitude to students and their parents who agreed to participate in this study. Also, we would like to thank the Ministry of Education, Palestine, for their support by facilitating communication with the schools and parents. Thanks also to all the co-researchers and fieldworkers involved in this study. Special thanks to the Nutrition and Food Technology Department, Faculty of Agriculture and Veterinary Medicine, An-Najah National University for the support given to researchers.

\section{Authors' contributions}

The authors have contributed to the manuscript as the following; Manal Badrasawi: the principal investigator has written the study proposal and protocol and supervised the data analysis. Ola Anabtawi: participated in the study protocol revision and wrote the first draft of the manuscript. Yaqout Al-Zain was in charge of research data management and data analysis. All authors have read and approved the final manuscript.

\section{Funding}

The authors declare that no external financial support was received for this study.

\section{Availability of data and materials}

The dataset used and analysed in this study is available from the corresponding author on reasonable request.

\section{Declarations}

\section{Ethics approval and consent to participate}

This project acquired ethical approval from the Institution review Board for Ethical approval from An-Najah National University. The research procedures were conducted in accordance with the principle expressed in the Declaration of Helsinki. The consent of participation was taken first from the school principals, then from the parents through the schoolteachers. Informed consent was obtained from all the parents and school principals before the data collection

\section{Consent for publication}

Not Applicable.

\section{Competing interests}

The authors declare they have no competing interests.

Received: 22 February 2021 Accepted: 22 June 2021

Published online: 06 August 2021

\section{References}

1. Aurino E, Fernandes M, Penny ME. The nutrition transition and adolescents diets in low- and middle-income countries: a cross-cohort comparison. Public Health Nutr. 2017;20(1):72-81. https://doi.org/10.1017/S136898001 6001865.

2. Lubbad T. Food consumption patterns and dietary habits associated with weight status in healthy young adult students. Gaza: Al Azhar University Gaza; 2011
3. Bi H, Gan Y, Yang C, Chen Y, Tong X, Lu Z. Breakfast skipping and the risk of type 2 diabetes: a meta-analysis of observational studies. Public Health Nutr. 2015;18(16):3013-9. https://doi.org/10.1017/S1368980015000257.

4. Watanabe Y, Saito I, Henmi I, Yoshimura K, Maruyama H, Yamauchi K, et al. Skipping breakfast is correlated with obesity. J Rural Med. 2014;9(2):51-8.

5. El Kishawi RR, Soo KL, Abed YA, Muda WAMW. Prevalence and predictors of overweight and obesity among women in the Gaza strip-Palestine: a crosssectional study. BMC Public Health. 2020;20(1):864. https://doi.org/10.1186/ s12889-020-08966-1.

6. Ellulu MS. Obesity, hypertension, and type-2 diabetes mellitus: the interrelationships and the determinants among adults in Gaza city, Palestine. Osong Public Health Res Perspect. 2018;9(6):289-98. https://doi. org/10.24171/j.phrp.2018.9.6.02.

7. Wesnes KA, Pincock C, Richardson D, Helm G, Hails S. Breakfast reduces declines in attention and memory over the morning in schoolchildren. Appetite. 2003;41(3):329-31. https://doi.org/10.1016/j.appet.2003.08.009.

8. On the occasion of the International Youth Day, the Palestinian Central Bureau of Statistics (PCBS) issues a press release demonstrating the situation of youth in the Palestinian society [http://www.pcbs.gov.ps/site/512/defa ult.aspx?lang=en\&ltemID=3787\#: :text=In\%20this\%20press\%20release\%2 C\%20the,(18\%2D29\%20years).\&text=

The\%20percentage\%20of\%20youth\%20(18,total\%20population\%20(1. $14 \% 20$ million)].

9. Currie C, Organization WH: Health behaviour in school-aged children (HBSC) study: international report from the 2001/2002 survey. 2004.

10. Mikki N, Abdul-Rahim HF, Shi Z, Holmboe-Ottesen G. Dietary habits of Palestinian adolescents and associated sociodemographic characteristics in Ramallah, Nablus and Hebron governorates. Public Health Nutr. 2010;13(9): 1419-29. https://doi.org/10.1017/S1368980010000662.

11. Abudayya AH, Stigum H, Shi Z, Abed Y, Holmboe-Ottesen G. Sociodemographic correlates of food habits among school adolescents (1215 year) in North Gaza strip. BMC Public Health. 2009:9(1):1-13.

12. Al-Hazzaa HM, Alhowikan AM, Alhussain MH, Obeid OA. Breakfast consumption among Saudi primary-school children relative to sex and socio-demographic factors. BMC Public Health. 2020;20:1-14.

13. Aguirre Velasco A, Cruz ISS, Billings J, Jimenez M, Rowe S. What are the barriers, facilitators and interventions targeting help-seeking behaviours for common mental health problems in adolescents? A systematic review. BMC Psychiatr. 2020;20(1):293. https://doi.org/10.1186/s12888-020-02659-0.

14. Al Sabbah H, Vereecken C, Kolsteren P, Abdeen Z, Maes L. Food habits and physical activity patterns among Palestinian adolescents: findings from the national study of Palestinian schoolchildren (HBSC-WBG2004). Public Health Nutr. 2007;10(7):739-46. https://doi.org/10.1017/S1368980007665501.

15. Al-Bashtawy M. Breakfast consumption habits among schoolchildren. J Pediatr Nurs. 2017:36:118-23. https://doi.org/10.1016/j.pedn.2017.05.013.

16. Ghrayeb FA, Al Rifai A: PUBLIC HEALTH. Prevalence of Lifestyle-Related Risk Factors Contributing to Non-Communicable Diseases among Adolescents in Tarqumia, Palestine. International Medical Journal 2014, 21(3):272-6.

17. Al-Hazzaa HM, Al-Rasheedi AA, Alsulaimani RA, Jabri L. Anthropometric, familial-and lifestyle-related characteristics of school children skipping breakfast in Jeddah, Saudi Arabia. Nutrients. 2020;12(12):3668. https://doi. org/10.3390/nu12123668

18. Golley RK, Maher CA, Matricciani L, Olds TS. Sleep duration or bedtime? Exploring the association between sleep timing behaviour, diet and BMI in children and adolescents. Int J Obes. 2013;37(4):546-51. https://doi.org/10.1 038/ijo.2012.212

19. Gong QH, Li H, Zhang XH, Zhang T, Cui J, Xu GZ. Associations between sleep duration and physical activity and dietary behaviors in Chinese adolescents: results from the youth behavioral risk factor surveys of 2015. Sleep Med. 2017:37:168-73. https://doi.org/10.1016/j.sleep.2017.06.024

20. Yang R-J, Wang EK, Hsieh Y-S, Chen M-Y. Irregular breakfast consumption and health status among adolescents in Taiwan. BMC Public Health. 2006; 6(1):295. https://doi.org/10.1186/1471-2458-6-295.

21. Keski-Rahkonen A, Kaprio J, Rissanen A, Virkkunen M, Rose RJ. Breakfast skipping and health-compromising behaviors in adolescents and adults. Eur I Clin Nutr. 2003:57(7):842-53. https://doi.org/10.1038/sj.ejcn.1601618.

22. Hassan AS, Al-Dosari SN. Breakfast habits and snacks consumed at school among Qatari schoolchildren aged 9-10 years. Nutri Food Sci. 2008;38(3): 264-70. https://doi.org/10.1108/00346650810871957.

23. Shaw ME. Adolescent breakfast skipping: an Australian study. Adolescence. 1998:33(132):851-61. 
24. Mehrabbeik A, Mahmoodabad SSM, Khosravi HM, Fallahzadeh H. Breakfast consumption determinants among female high school students of Yazd Province based on Pender's health promotion model. Electron Physician. 2017;9(8):5061-7. https://doi.org/10.19082/5061.

25. Reddan J, Wahlstrom K, Reicks M. Children's perceived benefits and barriers in relation to consumption breakfast in schools with or without universal school breakfast. J Nutr Educ Behav. 2002;34(1):47-52. https://doi.org/10.101 6/S1499-4046(06)60226-1.

26. Hearst MO, Shanafelt A, Wang Q, Leduc R, Nanney MS. Barriers, benefits, and behaviors related to breakfast consumption among rural adolescents. J Sch Health. 2016;86(3):187-94. https://doi.org/10.1111/josh.12367.

27. Mounayar R, Jreij R, Hachem J, Abboud F, Tueni M. Breakfast intake and factors associated with adherence to the Mediterranean diet among Lebanese high school adolescents. J Nutri Metab. 2019;2019:1-10. https:// doi.org/10.1155/2019/2714286.

\section{Publisher's Note}

Springer Nature remains neutral with regard to jurisdictional claims in published maps and institutional affiliations.

Ready to submit your research? Choose BMC and benefit from:

- fast, convenient online submission

- thorough peer review by experienced researchers in your field

- rapid publication on acceptance

- support for research data, including large and complex data types

- gold Open Access which fosters wider collaboration and increased citations

- maximum visibility for your research: over $100 \mathrm{M}$ website views per year

At BMC, research is always in progress.

Learn more biomedcentral.com/submissions 